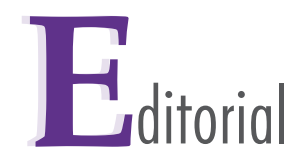

\title{
¿Puede la ciencia de datos ayudar a combatir la corrupción en la contratación pública?
}

\section{Generalidades}

Durante los últimos años ha habido un creciente interés por el uso y aplicación de las nuevas tecnologías asociadas a la cuarta revolución industrial. Hablar de macrodatos (big data o $B D$, por su nombre en inglés) e inteligencia artificial (IA) y de sus ventajas e innumerables usos prácticos se ha convertido en un referente conceptual de transformación en cualquier organización. Recurrentemente, se habla de diagnósticos médicos basados en redes neuronales artificiales, selección de factores de innovación a través de modelos de aprendizaje de máquina, asistentes virtuales en la atención de canales de mercadeo, predicción, control y patrones de velocidad en el transporte público urbano, entre otros. La lista de tecnologías y los escenarios futuros para su aplicación es interminable.

En este sentido, las ciencias de la computación proveen conceptos integradores que han permitido que los sistemas aprendan conductas, a través de algoritmos específicos y mediante el procesamiento de grandes volúmenes de datos. De esta manera, se encuentra que el fundamento primario de la IA es la generación y el ordenamiento de datos, conocido como BD, nervio motor de 
la IA. Con este manejo de los datos se busca recibir y procesar nuevas piezas de información, además de pronosticar y desarrollar tareas humanas repetitivas, rápida y eficientemente. Precisamente, este es uno de los principales paradigmas de la cuarta revolución industrial, pues es el desarrollo tecnológico lo que está impulsando el avance de la nueva era industrial en la cual estamos inmersos como humanidad.

En contraste, el uso que las personas no expertas hacen de los conceptos y potencialidades de estas nuevas tecnologías son, muchas veces, fuente de error y confusión, porque se abordan sin distinción, incluso como si fueran sinónimos, sin tener en cuenta que entre cada herramienta y cada concepto puede haber diferencias importantes, que sirven a su propio propósito y problemática específica de aplicación. Por esto, no obstante, no es de extrañar que dada la amplia variedad de conceptos para muchos apenas sea posible alcanzar una muy limitada comprensión conceptual de todo este campo.

Por ejemplo, el concepto de dato, cuya definición plana y simple indicaría algo cuyo valor es importante para personas, organizaciones o sistemas, ha generado avalanchas de discusiones éticas y legales en torno a su tratamiento.

Ahora bien, conceptos aún más complejos, como la ciencia de datos, constituyen la puerta de entrada hacia herramientas también más complejas.

La ciencia de datos se basa en la formulación de hipótesis sobre el mundo real y en el posterior análisis de los datos recopilados para validar o no dichas hipótesis. En la misma línea, se encuentra el aprendizaje de máquina, concepto que se sirve de los algoritmos para ayudar a un sistema computacional a adquirir conocimiento mediante el uso de datos. Este concepto es, a su vez, complementario al de ciencia de datos y, por eso, es adecuado afirmar que el aprendizaje de maquina puede ser, y a menudo es, parte de una adecuada ciencia de datos, que es fundamentalmente un proceso, mientras que el aprendizaje de maquina es una herramienta que puede ser inmensamente útil para llevar a cabo este proceso. Finalmente, atravesando toda la red de conceptos, se encuentra la IA, cuya aplicación se ha centrado en lograr que las máquinas imiten la inteligencia humana. 


\section{A grandes males, grandes remedios}

El uso extensivo de estas tecnologías ha ido encontrando nuevos y potenciales nichos de aplicación que, al mismo tiempo, han incluido dificultades y controversias muy recurrentes, y que son fuente de preocupación para nuestra sociedad. Un par de esos males son la corrupción y el fraude que, aunque no son nuevos, sí han encontrado en el uso inexistente de la tecnología y en el desconocimiento de la gente una manera de mantenerse vigentes.

La corrupción pública es el abuso malintencionado de una posición de poder para adquirir beneficios materiales en cualquier organización o institución del Estado. Las diferentes formas de corrupción provocan múltiples impactos y son extensos los factores que contribuyen a su existencia. Pero en particular, la corrupción inhibe el crecimiento económico, afectando negocios, empleos e inversiones, lo que deriva en una disminución de la calidad de vida y un aumento de la desconfianza en el Estado, porque nos priva a todos de oportunidades. En general, la corrupción es un fenómeno sistémico, complejo y adaptativo que requiere de enfoques multidisciplinarios para su correcta detección y combate.

Uno de los casos más recientes de corrupción se hizo visible con la contingencia producida por la COVID-19, cuyo efecto se sintió no solamente en el bienestar de los individuos, sino también en los sistemas de salud, donde actores inescrupulosos han aprovechado para robar por medio de contrataciones públicas a través de plataformas digitales del Estado. Es bien sabido que, en muchos casos, el manejo ineficiente de la contratación pública la hace vulnerable a la corrupción. Esto se debe principalmente a factores como estructuras rígidas, falta de articulación entre el gobierno y las entidades, manejo ineficiente de tiempo y dinero y monitoreo limitado o inexistente.

Por fortuna, toda esta situación ha llevado también a pensar en mecanismos de acción, detección y prevención para levantar alertas o banderas rojas sobre aquellas acciones presumiblemente fraudulentas o no éticas para evitar que se repitan. La pregunta que surge de esta problemática es ¿de qué manera se puede hacer un uso efectivo y eficiente de las nuevas tecnologías computacionales para prevenir de forma categórica el flagelo del fraude y corrupción en procesos de contratación estatales? 
EDITORIAL

\section{Ciencia, tecnología e innovación como respuesta}

Albert Einstein sabiamente acuñó la frase «Locura es hacer lo mismo una y otra vez esperando obtener resultados diferentes». Dicha frase debería motivarnos a iniciar el camino del cambio, del pensamiento divergente que desemboque en aproximaciones pragmáticas inexploradas; en otras palabras, en innovación aplicada a problemas que tradicionalmente se han abordado con metodologías rígidas o tradicionalistas.

En este caso, involucrar la ciencia y el análisis de datos como motor de innovación para transformar la contratación publica, generando mecanismos para combatir la corrupción en el Estado, ha resultado ser una solución no solo factible, sino además aprovechable desde múltiples problemáticas. Algunos trabajos y experiencias que dan cuenta de estas soluciones incluyen el análisis exploratorio de datos en contrataciones en la prestación de servicios de salud; el uso del procesamiento de lenguaje natural para el análisis de contratos de vacunación en pandemia; la detección de patrones de fraude en contratos públicos, utilizando aprendizaje no supervisado y algoritmos de clusterización, entre otros.

En general, la creciente atención que se dirige a este sector y el esfuerzo innovador han permitido que múltiples actores de la academia, investigadores, profesionales en diversas áreas, como los gestores tecnológicos, y el sector privado converjan hacia formas abiertas de innovación y se unan en un objetivo común, haciendo uso de datos abiertos, como la plataforma del Sistema Electrónico para la Contratación Pública (SECOP), que en Colombia es la herramienta de que dispone el gobierno para informar sobre toda la contratación que se hace con el dinero del erario público. Asimismo, se pueden mencionar diversas metodologías que incluyen la administración de proyectos, agilismo, Scrum, CRISP-DM, entre otras.

Esto ha generado un caudal ascendente de nuevas y mejoradas aplicaciones, muchas de las cuales se encuentran en etapa de propuesta, desarrollo, o mejoramiento, todo con el fin de aportar una solución a esta gravísima problemática social. 


\section{Desafíos para el futuro}

El futuro requiere propuestas a la altura de los desafíos. La detección de la corrupción en la contratación no es un reto puramente académico o empresarial, es un compromiso de sociedad que involucra a todos los sectores y a cada uno de los ciudadanos. Por lo tanto, si se quiere mejorar se deben adoptar enfoques innovadores y el Gobierno debe acompañar a la sociedad en su implementación; para que las estrategias de mejoramiento se articulen con las necesidades de la ciudadanía y se debe procurar que los marcos de control conviertan a la gente y a las organizaciones sociales en aliados en el desarrollo de propuestas de ciencia de datos para la identificación de riesgos o posibles hechos de corrupción, lo cual, en última instancia, fortalecería la corresponsabilidad entre el sector público y el privado.

\section{ID) Julián Alberto Uribe-Gómez}

julianuribe@itm.edu.co

MSc en gestión tecnológica - científico de datos Docente Facultad de Ciencias Económicas y Administrativas Instituto Tecnológico Metropolitano - ITM 